\title{
Where is the varicella vaccine?
}

$\mathrm{E}$ arly in 1995, the first live, attenuated varicella zoster virus (VZV) vaccine (Varivax, Merck) was licensed in the United States. The promotional activities for this vaccine have been noticed by Canadian parents, physicians, susceptible health care workers and members of the media, leading to a chorus of questions about when a VZV vaccine will be licensed in this country. The short answer is, not soon. Of the three companies able to manufacture vaccines based on the Oka attenuated VZV strain, none has applied to license a product in Canada. Moreover, no manufacturer has been willing to make this vaccine available to individual patients through the Emergency Release Program of Health Canada. Thus, the only option for patients who want to be vaccinated now is to attend a provider in the United States and pay the substantial costs involved. This situation is unlikely to change in less than 18 to 24 months.

What explains the delay in manufacturers seeking to license VZV vaccines in Canada? One can only surmise that there are various constraints on suppliers, but some factors are obvious enough. For Merck, the main challenge is to supply a rapidly growing domestic market. During the decadelong development period of Varivax, advisory groups had ample time to consider the appropriate uses of the vaccine, estimate its cost effectiveness and have their concerns about vaccine safety and effectiveness addressed by numerous studies. Consequently, both the American Academy of Pediatrics and the Advisory Committee on Immunization Practices of the United States Public Health Service were prepared from the outset to endorse universal vaccination of infants at 12 to 18 months of age and recommend catch-up vaccination of susceptible older children and adolescents. The vaccine was added to the federal Vaccines for Children Program, ensuring its availability to recipients of subsidized health care. By the end of 1995, over 2 million doses were distributed. As more physicians become convinced of the advantages of varicella vaccination and the federal purchase contract begins, sales will increase substantially in 1996. It could be a year or two before domestic

All material presented in Pediatric Infectious Disease Notes has been reviewed and approved by the chairperson, Canadian Paediatric Society Board and representative members of the Canadian Paediatric Society Committee on Infectious Diseases and Immunization

Correspondence: Infectious Diseases and Immunization Committee, Canadian Paediatric Society, 401 Smyth Road, Ottawa, Ontario K1H 8L1. Telephone 613-737-2728, fax 613-737-2794 demand stabilizes and the manufacturer is able to determine whether sufficient supply exists to serve additional foreign markets. The other two manufacturers may be facing similar expectations to supply their European home markets first.

To serve the Canadian market appropriately, a manufacturer should be able to supply enough VZV vaccine for $80 \%$ or more of children for whom it is recommended. Unlike most new vaccines for which some use is better than none, varicella vaccine requires a full embrace. Without $80 \%$ or greater uptake of vaccine, mathematical models indicate that serious morbidity from wild varicella will actually increase as a result of continuing transmission and a shift in cases to older children and adolescents in whom illness is more severe. This also means that providers (ie, provincial ministries of health) should commit to universal varicella vaccination at a convenient time following licensure. Realistically, provinces are facing higher priorities for vaccine funding just now in coping with measles eradication, hepatitis B control programs and replacement of whole with acellular pertussis vaccines. When those programs are in place, varicella could be the next priority. Perhaps when provinces are able to commit to universal programs, combination vaccines with measles, mumps, rubella and varicella components will be available to facilitate them. With the Merck VZV vaccine, greater temperature stability is needed to make a combination vaccine practicable. For the other manufacturers, whose VZV vaccine is stable at $2^{\circ} \mathrm{C}$ to $8^{\circ} \mathrm{C}$, the challenge is to find a mumps vaccine that is acceptably safe in the United States and Canada.

Among the barriers to acceptance of VZV vaccine have been concerns about the unknowns, ie, the possibility of rare but severe adverse events, duration of protection, etc. By the time Varivax is licensed in Canada, safety data will be available from large-scale postmarketing studies in the United States involving infants and adolescents. Follow-up studies of the duration of protection will also have been extended. Lessons will have been learned regarding distribution and storage of this unstable product. The current requirement to keep it frozen at $-15^{\circ} \mathrm{C}$ or colder until just before use would mean that most vaccine providers in Canada would not be able to keep it in stock because few have such powerful freezers. In short, by accessing varicella vaccine later, we can avoid or minimize some of the 'pioneering' problems being encountered in the United States.

While it is unusual for Canada to be assigned observer status at the birth of a new vaccine, adopting it later has some advantages. Many of the concerns regarding a brand-new vaccine will have been resolved. Timing may be better suited 
for provinces to fund its universal use. Practical application of the vaccine may be enhanced by availability of thermostable products or combinations with measles-mumps-rubella vaccine. On balance, I believe that the trade-offs that accompany delayed availability of VZV vaccine will be seen as being advantageous by providers and consumers.
David W Scheifele MD Vaccine Evaluation Center $B C$ 's Children's Hospital and the Department of Pediatrics University of British Columbia Vancouver, British Columbia 


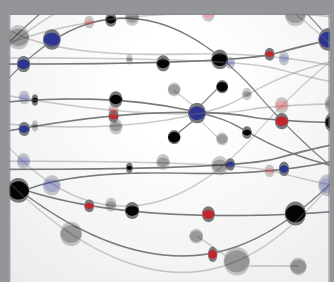

The Scientific World Journal
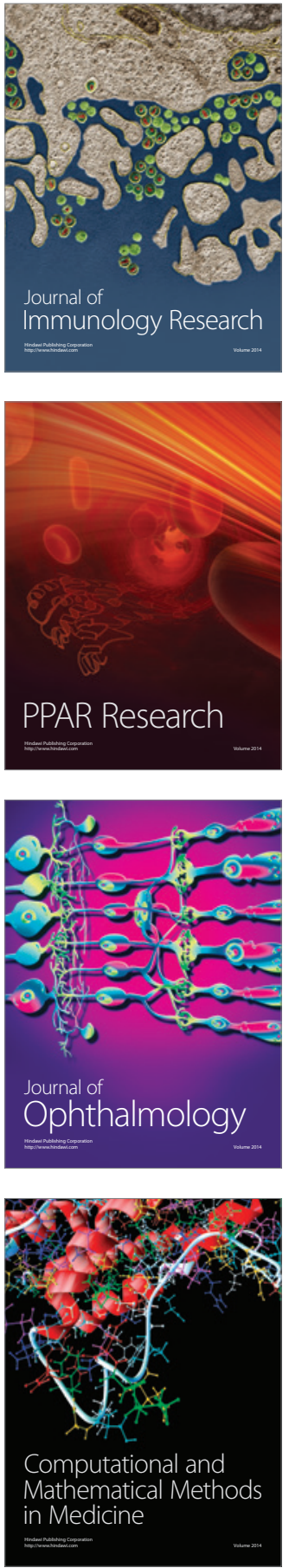

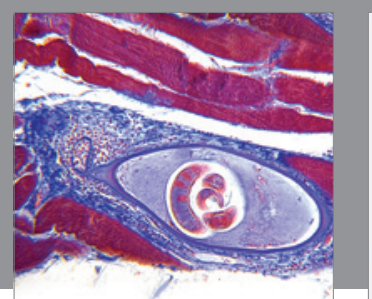

Gastroenterology Research and Practice

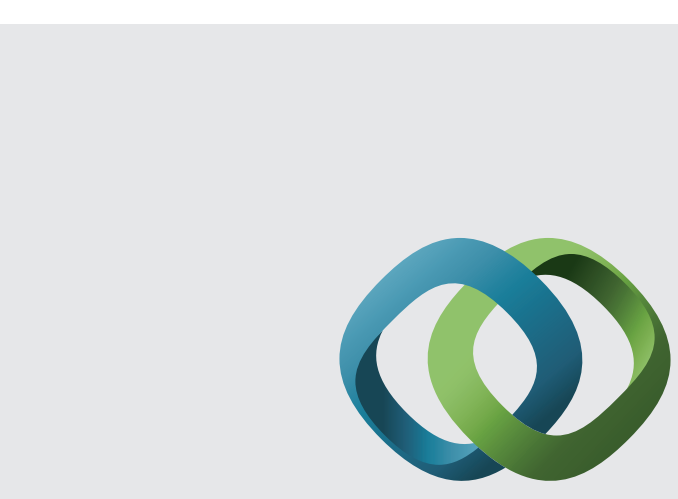

\section{Hindawi}

Submit your manuscripts at

http://www.hindawi.com
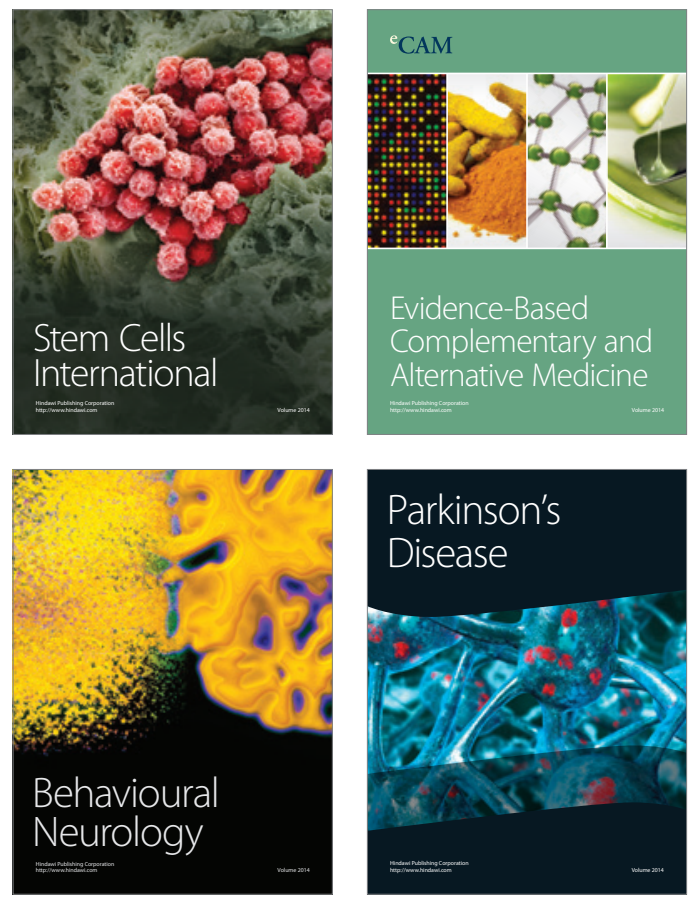
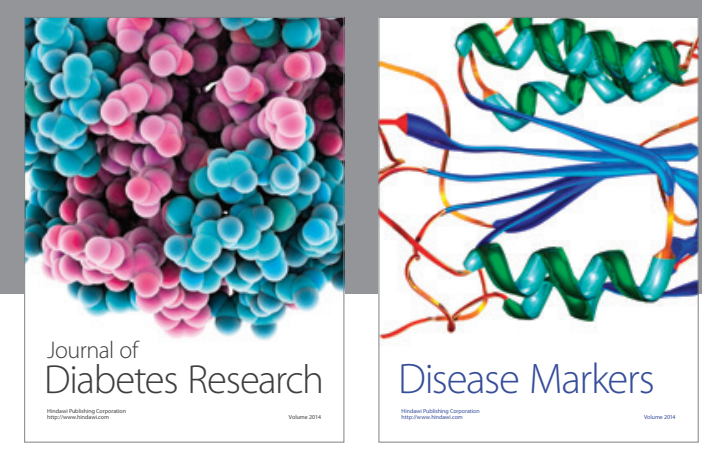

Disease Markers
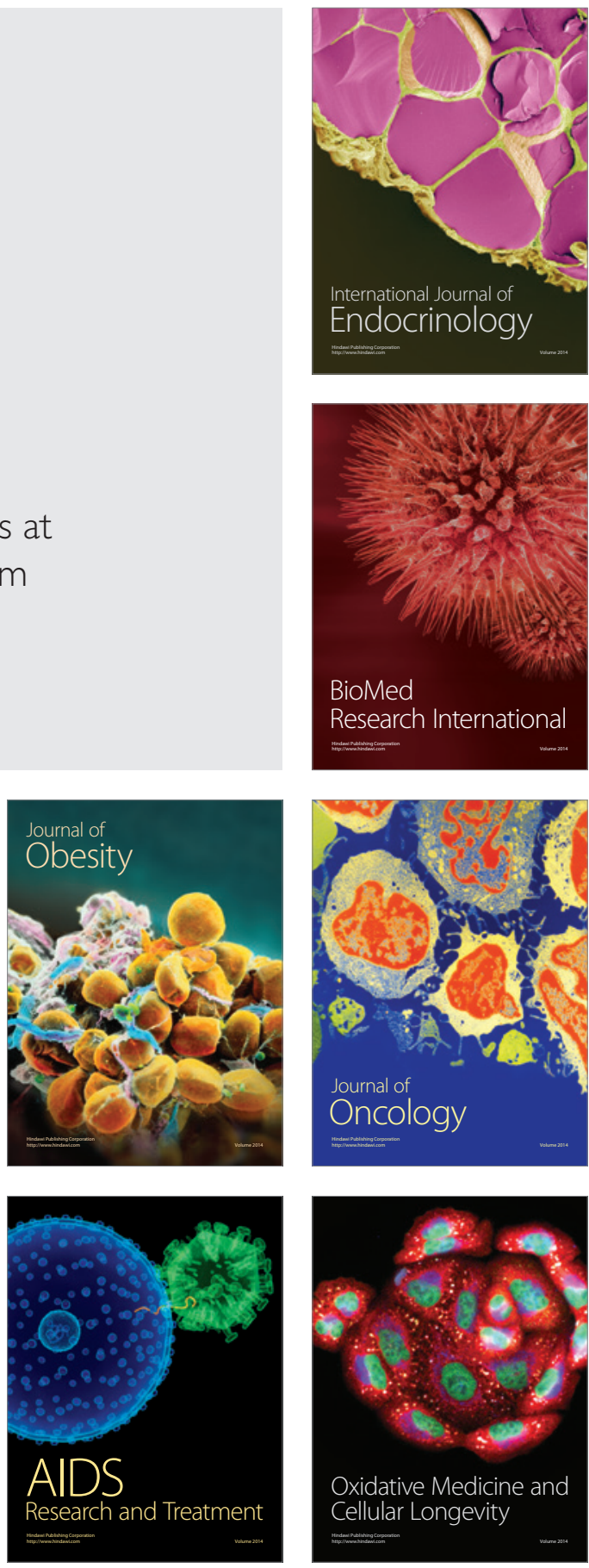